TRANSACTIONS OF THE

AMERICAN MATHEMATICAL SOCIETY

Volume 348, Number 10, October 1996

\title{
GROSS SPACES
}

\author{
SAHARON SHELAH AND OTMAR SPINAS
}

\begin{abstract}
A Gross space is a vector space $E$ of infinite dimension over some field $F$, which is endowed with a symmetric bilinear form $\Phi: E^{2} \rightarrow F$ and has the property that every infinite dimensional subspace $U \subseteq E$ satisfies $\operatorname{dim} U^{\perp}<\operatorname{dim} E$. Gross spaces over uncountable fields exist (in certain dimensions) (see $[\mathrm{G} / \mathrm{O}]$ ). The existence of a Gross space over countable or finite fields (in a fixed dimension not above the continuum) is independent of the axioms of ZFC. This was shown in $[\mathrm{B} / \mathrm{G}],[\mathrm{B} / \mathrm{Sp}]$ and $[\mathrm{Sp} 2]$. Here we continue the investigation of Gross spaces. Among other things, we show that if the cardinal invariant $\mathbf{b}$ equals $\omega_{1}$, a Gross space in dimension $\omega_{1}$ exists over every infinite field, and that it is consistent that Gross spaces exist over every infinite field but not over any finite field. We also generalize the notion of a Gross space and construct generalized Gross spaces in ZFC.
\end{abstract}

\section{INTRODUCTION}

Let $E$ be a vector space of infinite dimension over some field $F$, and let $\Phi$ : $E \times E \rightarrow F$ be a symmetric bilinear form. If $U$ is a subspace of $E$, the orthogonal complement is denoted by $U^{\perp}$. If $U$ has finite dimension, then clearly we have $\operatorname{codim}_{E} U^{\perp} \leq \operatorname{dim} U$, where $\operatorname{codim}_{E} U^{\perp}$ is the dimension of some linear complement of $U^{\perp}$ in $E$. As is well-known from Hilbert space, this is completely false if $U$ is of infinite dimension.

In $[\mathrm{G} / \mathrm{O}]$, the investigation of quadratic spaces sharing the following strong property has been started:

(*) for all subspaces $U \subseteq E$ of infinite dimension: $\operatorname{dim} U^{\perp}<\operatorname{dim} E$.

Such a space we call a Gross space. In [G/O], the motivation for this was that Gross spaces are natural candidates to have a small orthogonal group in the sense that every isometry is the product of finitely many hyperplane reflections. If a Gross space is the orthogonal sum of two subspaces, then one of them must be finite dimensional. Hence, a Gross space is far from having an orthogonal basis, and so its dimension must be uncountable.

In $[\mathrm{G} / \mathrm{O}]$, over every uncountable field $F$ a strong Gross space has been constructed, i.e. a space sharing the following stronger property:

(**) for all subspaces $U \subseteq E$ of infinite dimension: $\operatorname{dim} U^{\perp} \leq \aleph_{0}$.

Such a construction has been achieved in every uncountable dimension less than or equal to the cardinality of the field.

Constructing a Gross space gets more difficult the smaller the cardinality of the field is compared with the dimension of the space.

Received by the editors August 1, 1995.

1991 Mathematics Subject Classification. Primary 11E04, 03E35; Secondary 12L99, 15A36.

The authors are supported by the Basic Research Foundation of the Israel Academy of Science.

(C)1996 American Mathematical Society 
In $[\mathrm{B} / \mathrm{G}]$, a Gross space of dimension $\aleph_{1}$ (so Gross=strongly Gross) has been constructed over every countable or finite field. But for this the Continuum Hypothesis $(\mathrm{CH})$ has been assumed, and hence the question was raised whether $\mathrm{CH}$ is necessary or if a construction in ZFC is possible.

In [Sp1], [B/Sp] and [Sp2], it turned out that this question leads into set theory and that in fact it is independent of ZFC.

Here we answer several open questions from these papers and we also continue the investigation of Gross spaces over uncountable fields in $[\mathrm{G} / \mathrm{O}]$.

A difficult result in $[\mathrm{B} / \mathrm{Sp}]$ says that if $\mathbf{b}=\omega_{1}$, then a Gross space of dimension $\aleph_{1}$ exists over every field which is the extension of some finite or countable field by countably many transcendentals. Here $\mathbf{b}$ is defined as the minimal cardinality of a family of functions from the natural numbers to themselves which is unbounded under eventual dominance. Clearly $\omega_{1} \leq \mathbf{b} \leq \mathbf{c}$. But ZFC does not decide where $\mathbf{b}$ lies exactly. In $\S 2$ we will show that if $\mathbf{b}=\omega_{1}$, then a Gross space of dimension $\aleph_{1}$ exists over every infinite field.

In $\S 3$ we show that $\mathbf{b}=\omega_{1}$ may hold but no symmetric bilinear space of dimension $\aleph_{1}$ over any finite field is a Gross space. We prove three variants of this. The first uses a forcing from [Sp2] which kills Gross spaces, the second involves the splitting number $\mathbf{s}$, and the third uses a model from [B/Sh] where simultaneously $P_{\aleph_{1}}$ - and $P_{\aleph_{2}}$-points exist. In this model there exist no Gross spaces over finite fields at all.

For a couple of years the main open problem about Gross spaces has been whether there exists a ZFC model where there exists no Gross space over any countable or finite field in any dimension. The first author thinks that he has constructed a model for this, using a new iteration technique. See [Sh 538] in Shelah's list of publications.

In $\S 4$ we investigate a natural generalization of the Gross property:

$(* * *)$ for all subspaces $U \subseteq E$ of dimension $\geq \lambda$ : $\operatorname{dim} U^{\perp}<\operatorname{dim} E$.

Here $\lambda$ is an infinite cardinal less than or equal to the dimension of $E$. A space sharing the property $(* * *)$ is called a $\lambda$-Gross space. Hence a Gross space is an $\omega$-Gross space.

We concentrate on the results in ZFC. We show that $\omega_{1}$-Gross spaces of dimension $\aleph_{1}$ and $\aleph_{2}$ always exist over every countably infinite field. We also show that $|F|^{\lambda}$ is an upper bound for the dimension of a $\lambda$-Gross space over $F$. Hence, in ZFC these results are maximal. We do not know whether these results hold also for finite fields. But we show that if $\lambda>\omega_{1}$ is regular uncountable, then a $\lambda^{+}$-Gross space of dimension $\lambda^{+}$exists over any field.

We also investigate the situation for uncountable fields. The construction in $[\mathrm{G} / \mathrm{O}]$ yields strong Gross spaces of dimension at most as large as the cardinality of the base field. Is it possible to enlarge the dimension of the space while keeping the size of the field small? Over every field of uncountable cardinality $\lambda$ we construct a $\lambda$-Gross space of dimension $\lambda^{+}$, as well as a $\lambda^{+}$-Gross space of dimension $\lambda^{++}$. Again, by the upper bound for the dimension of a $\lambda$-Gross space mentioned above, these results are maximal in ZFC.

We remark that all the investigations mentioned above may take place in a rather more general context. First, everything remains true if we skip to orthosymmetric sesquilinear forms over a division ring endowed with an involutory antiautomorphism (see $[G]$ for the definitions). Second, using a representation theorem for AC-lattices equipped with a polarity, the results can be transferred to the level of abstract ortho-lattices. This has been announced in [Sp3]. 
For a survey on the whole subject refer to [Sp4].

\section{Notation AND DEFINITIONS}

\subsection{Forms.}

Let $F$ be a commutative field of arbitrary characteristic. Let $E$ be a vector space over $F$, endowed with a symmetric bilinear form $\Phi: E \times E \rightarrow F$, i.e. $\Phi$ is linear in both arguments and $\Phi(x, y)=\Phi(y, x)$ always. Most of the spaces we construct will have isotropic vectors, i.e. nonzero vectors $x$ such that $\Phi(x, x)=0$. For a subspace $U \subseteq E$ the orthogonal complement $U^{\perp}$ is the subspace $\{x \in E: \forall y \in U$ $\Phi(x, y)=0\}$. We call $\langle E, \Phi\rangle$ nondegenerate if $E^{\perp}=\{0\}$. By $E^{*}$ we denote the $F$ vectorspace of linear functionals $E \rightarrow F$. If in $E$, we have fixed a basis $\left\langle e_{\alpha}: \alpha \in I\right\rangle$, then for a vector $x \in E$ the support of $x$, denoted by $\operatorname{supp}(x)$, is the unique finite set of $\alpha$ 's such that, in the representation of $x$ by $\left\langle e_{\alpha}: \alpha \in I\right\rangle, e_{\alpha}$ has a nonzero coefficient.

\subsection{Set theory.}

For the theory of forcing refer to $[\mathrm{B}, \mathrm{J}, \mathrm{K}]$ or $[\mathrm{Sh} 1]$.

For sets $A, B$, the set of functions from $A$ to $B$ is denoted by ${ }^{A} B$. If $\kappa, \lambda$ are cardinals, then $\kappa^{\lambda}$ denotes the cardinality of the set ${ }^{\lambda} \kappa$, and $[A]^{\lambda}$ denotes the set of all subsets of $A$ which have cardinality $\lambda$. By $\mathbf{c}$ we denote the cardinality of the continuum. If $A$ is well-ordered, then o.t. $(A)$ is its order type.

If $f, g \in{ }^{\omega} \omega$, then we say $f$ eventually dominates $g$ and we write $g<^{*} f$, if $\exists k \forall n \geq k g(n)<f(n)$. A family $F$ of members of ${ }^{\omega} \omega$ is $<^{*}$-unbounded if there is no $f \in{ }^{\omega} \omega$ such that $\forall g \in \operatorname{ran} F g<^{*} f$. $F$ is called dominating if every function from ${ }^{\omega} \omega$ is eventually dominated by some member of $F$.

Then $\mathbf{b}$ is defined as the minimal cardinality of a $<^{*}$-unbounded family in ${ }^{\omega} \omega$.

The cardinal invariant $\mathbf{d}$ is defined as the minimal cardinality of a dominating family.

For $A, B \in[\omega]^{\omega}$, we write $A \subseteq^{*} B$ if $A \backslash B$ is finite. A family $\mathcal{S}$ of members of $[\omega]^{\omega}$ is called a splitting family, if for every $A \in[\omega]^{\omega}$ there exists $S \in \operatorname{ran} \mathcal{S}$ such that $A \cap S$ and $A \backslash S$ are both infinite.

The splitting number $\mathbf{s}$ is defined as the minimal cardinality of a splitting family.

Here $\mathbf{b}, \mathbf{d}$ and $\mathbf{s}$ are instances of so called cardinal invariants of the continuum which have found lots of applications throughout mathematics. See [vD] for an introduction.

The following theorems form part of the folklore in set theory. Proofs of the first two of them may be found in $[\mathrm{vD}]$.

Theorem $\mathbf{1}$. $\mathbf{b}, \mathbf{d}$ and $\mathbf{s}$ are uncountable cardinals $\leq \mathbf{c} ; \mathbf{b}, \mathbf{s} \leq \mathbf{d}$, and $\mathbf{b}$ is regular.

The axioms of ZFC do not decide where exactly $\mathbf{b}, \mathbf{d}$ and $\mathbf{s}$ lie.

Theorem 2. Let $\kappa$ and $\lambda$ be regular cardinals with $\omega_{1} \leq \kappa \leq \lambda$. There exists a forcing preserving all cardinals such that in the extension obtained by forcing with $i t, \mathbf{c}=\lambda$ and $\mathbf{b}=\mathbf{d}=\mathbf{s}=\kappa$ hold.

Theorem $3[\mathrm{Sh} 2] /[\mathrm{B} / \mathrm{S}] \cdot \omega_{1}=\mathbf{b}<\mathbf{s}$ is consistent with ZFC as well as is $\omega_{1}=$ $\mathbf{s}<\mathbf{b}$. 


\section{When $\mathbf{b}=\omega_{1}$, A Gross SPACE EXISTS OVER EVERY INFINITE FIELD}

In $[\mathrm{B} / \mathrm{Sp}, \S 4]$ it has been shown that the assumption $\mathbf{b}=\omega_{1}$ implies that a Gross space of dimension $\omega_{1}$ exists over any field which is the extension of an arbitrary finite or countable field by countably many transcendentals. Here we show that $\mathbf{b}=\omega_{1}$ is enough to construct a Gross space of dimension $\omega_{1}$ over any countably infinite field. In $\S 3$, we will show that $\mathbf{b}=\omega_{1}$ may hold but no Gross spaces exist over finite fields.

The construction will use the filtrations given by the following Lemma.

Lemma. There exists a family $\left\langle A_{k}^{\alpha}: \alpha<\omega_{1}, k<\omega\right\rangle$ of finite sets such that for any $\alpha, \beta<\omega_{1}$ and $k, l<\omega$ the following requirements are satisfied:

(1) $\alpha=\bigcup_{k<\omega} A_{k}^{\alpha}$,

(2) if $k<l$, then $A_{k}^{\alpha} \subseteq A_{l}^{\alpha}$,

(3) if $\beta<\alpha$ and $\beta \in A_{k}^{\alpha}$, then $A_{k}^{\beta}=A_{k}^{\alpha} \cap \beta$.

Proof. We define $A_{k}^{\alpha}$ by induction on $\alpha$.

For $\alpha=0$, let $A_{k}^{\alpha}=\emptyset$.

For $\alpha=\beta+1$, let $A_{k}^{\alpha}=A_{k}^{\beta} \cup\{\beta\}$.

For $\alpha$ a limit, let $\left\langle\alpha_{n}: n<\omega\right\rangle$ be an increasing sequence such that $\alpha=$ $\sup _{n<\omega} \alpha_{n}$. Now choose $\left\langle k_{n}: n<\omega\right\rangle$ increasing such that for all $n$ we have $\alpha_{n} \in A_{k_{n}}^{\alpha_{n+1}}$. Define

$$
A_{m}^{\alpha}=A_{m}^{\alpha_{0}}
$$

for $m \leq k_{0}$ and

$$
A_{m}^{\alpha}=A_{m}^{\alpha_{l+1}}
$$

for $k_{l}<m \leq k_{l+1}$. It is easy to check that this works.

Theorem. Assume $\mathbf{b}=\omega_{1}$. Then a Gross space of dimension $\aleph_{1}$ exists over every infinite field.

Proof. Let $F$ be a countable, infinite field, and let $E$ be a vector space over $F$, spanned by a basis $\left\langle e_{\alpha}: \alpha<\omega_{1}\right\rangle$.

Let $\left\langle p_{n}: n \in \omega\right\rangle$ enumerate all polynomials in finitely many variables with coefficients in $F$.

In $F$ choose $\left\langle a_{n}: n<\omega\right\rangle$ such that for any polynomial $p\left[X_{1}, \ldots, X_{k}\right]$, for any $n$ larger than some integer depending on $p$ we have $a_{n} \notin\left\{p\left(a_{l_{1}}, \ldots, a_{l_{k}}\right): l_{1}, \ldots, l_{k}<\right.$ $n\}$.

Finally, let $\left\langle f_{\alpha}: \alpha<\omega_{1}\right\rangle$ be an unbounded, well-ordered family of increasing functions in $\omega^{\omega}$.

For any $\alpha<\omega_{1}$ we define a function $h_{\alpha}: \alpha \rightarrow \omega$ by induction, using the filtrations from the Lemma. Assume $\gamma \in A_{k+1}^{\alpha} \backslash A_{k}^{\alpha}$ and assume $h_{\beta}$ for $\beta<\alpha, h_{\alpha} \uparrow A_{k}^{\alpha}$ and $h_{\alpha} \uparrow\left(A_{k+1}^{\alpha} \cap \gamma\right)$ have been defined.

Let $h_{\alpha}(\gamma)=n$ such that $a_{n}$ is distinct from all the finitely many scalars of the form

$$
p_{i}\left(a_{l_{1}}, a_{l_{2}}, \ldots\right)
$$

where $i<f_{\alpha}(k+1)$ and any $l_{j}$ belongs to the range of one of the following functions: $h_{\alpha} \uparrow A_{k}^{\alpha}, h_{\alpha} \uparrow\left(A_{k+1}^{\alpha} \cap \gamma\right), h_{\beta} \uparrow A_{k+1}^{\beta}$ for $\beta \in A_{k+1}^{\alpha}$. 
Now define a symmetric bilinear form $\Phi: E \times E \rightarrow F$ as follows. For $\alpha<\beta<\omega_{1}$ define:

$$
\Phi\left(e_{\alpha}, e_{\beta}\right)=a_{n} \text { if and only if } h_{\beta}(\alpha)=n \text {. }
$$

$\Phi\left(e_{\beta}, e_{\beta}\right)$ may be defined arbitrarily.

We have to show that $\langle E, \Phi\rangle$ is Gross. Assume that this is not true. So there is a subspace $U$ of infinite dimension, spanned by a basis $\left\langle y_{k}: k<\omega\right\rangle$, such that $\operatorname{dim} U^{\perp}$ is uncountable. Each $y_{k}$ has a representation

$$
y_{k}=\sum_{l=1}^{m_{k}} b_{k l} e_{\alpha(k, l)}
$$

with nonzero coefficients $b_{k l}$. Choose $\alpha^{*}<\omega_{1}$ such that $U \subseteq \operatorname{span}\left\langle e_{\alpha}, \alpha<\alpha^{*}\right\rangle$. Using a $\Delta$-system argument and the bilinearity of the form, in $U^{\perp}$ we may certainly find vectors $\left\langle z_{\iota}, \iota<\omega_{1}\right\rangle$, all of them having the same nonzero coefficients in their representation, say

$$
z_{\iota}=\sum_{l=1}^{n} c_{l} e_{\beta(\iota, l)}
$$

such that for all $\iota_{1}<\iota_{2}<\omega_{1}$ we have $\alpha^{*}<\beta\left(\iota_{1}, 1\right)<\cdots<\beta\left(\iota_{1}, n\right)<\beta\left(\iota_{2}, 1\right)<$ $\cdots<\beta\left(\iota_{2}, n\right)$. Furthermore, we may assume that for some $k^{*}<\omega$, for all $\iota<\omega_{1}$, $1 \leq l_{1}<l_{2} \leq n$ and $1 \leq l \leq n$ we have

$$
\beta\left(\iota, l_{1}\right) \in A_{k^{*}}^{\beta\left(\iota, l_{2}\right)} \text { and } \alpha^{*} \in A_{k^{*}}^{\beta(\iota, l)}
$$

Since $\left\langle f_{\alpha}, \alpha<\omega_{1}\right\rangle$ is unbounded and well-ordered there exists $j<\omega$ such that $\left\langle f_{\beta(\iota, n)}(j), \iota \in \omega_{1}\right\rangle$ is unbounded in $\omega$. Since the $f_{\alpha}$ are increasing, this remains true if $j$ is replaced by any $j^{\prime} \geq j$.

Now choose $k<\omega$ such that the minimal $j^{\prime}$ with the property

$$
\left\{\alpha(k, 1), \ldots, \alpha\left(k, m_{k}\right)\right\} \subseteq A_{j^{\prime}+1}^{\alpha^{*}}
$$

is larger than $\max \left\{j, k^{*}\right\}$. Note that by (1) and property (3) of the filtrations from the Lemma, $j^{\prime}$ is minimal such that $(2)$ holds for any $\beta(\iota, l)$ instead of $\alpha^{*}$.

Choose $l_{0} \in\left\{1, \ldots, m_{k}\right\}$ such that $\alpha\left(k, l_{0}\right)$ is maximal in $A_{j^{\prime}+1}^{\alpha^{*}} \backslash A_{j^{\prime}}^{\alpha^{*}}$. Then the polynomial

$$
\sum_{\left\langle l, l^{\prime}\right\rangle \in\{1, \ldots, n\} \times\left\{1, \ldots, m_{k}\right\} \backslash\left\langle n, l_{0}\right\rangle} b_{k l_{0}}^{-1} c_{n}^{-1} b_{k l^{\prime}} c_{l} X_{l l^{\prime}}
$$

has its number, say $i$, in the enumeration of all polynomials fixed in the beginning. There exists $\iota<\omega_{1}$ such that $f_{\beta(\iota, n)}\left(j^{\prime}+1\right)>i$. From the definition of $\Phi$, using (1), we conclude

$$
\Phi\left(e_{\beta(\iota, n)}, e_{\alpha\left(k, l_{0}\right)}\right) \neq-\sum_{\left\langle l, l^{\prime}\right\rangle \in\{1, \ldots, n\} \times\left\{1, \ldots, m_{k}\right\} \backslash\left\langle n, l_{0}\right\rangle} b_{k l_{0}}^{-1} c_{n}^{-1} b_{k l^{\prime}} c_{l} \Phi\left(e_{\beta(\iota, l)}, e_{\alpha\left(k, l^{\prime}\right)}\right) .
$$

But then $\Phi\left(z_{\iota}, y_{k}\right) \neq 0$, a contradiction. 


\section{Gross SPACES OVER FINITE FIELDS}

The first Lemma shows that $\mathbf{c}$ is an upper bound for the dimension of a Gross space over finite or countable fields.

Lemma 1. Let $\langle E, \Phi\rangle$ be a symmetric bilinear space over $F$, and let $\lambda$ be an infinite cardinal $\leq$ the dimension of $E$. If $\langle E, \Phi\rangle$ has the property

$(* * *)$ for all subspaces $U \subseteq E$ of dimension $\geq \lambda$ : $\operatorname{dim} U^{\perp}<\operatorname{dim} E$.

Proof. Let $\operatorname{dim} E=\kappa$ and let $\left\langle e_{\alpha}: \alpha<\kappa\right\rangle$ be a basis. For $\alpha<\kappa$ define

$$
f_{\alpha}: \lambda \rightarrow F, \beta \mapsto \Phi\left(e_{\alpha}, e_{\beta}\right) .
$$

We get $\kappa=\bigcup_{f \in \mathcal{\lambda}_{F}} A_{f}$, where $A_{f}=\left\{\alpha<\kappa: f_{\alpha}=f\right\}$ and hence $\kappa=$ $\sum_{f \in \in_{F} F}\left|A_{f}\right|$.

So if $\kappa>|F|^{\lambda}$ there exists $B \subseteq{ }^{\lambda} F$ such that $\sum_{f \in B}\left|A_{f}\right|=\kappa$ and $\left|A_{f}\right| \geq 2$, for all $f \in B$. Fix $\alpha_{f} \in A_{f}$ for $f \in B$. We conclude that

$$
\bigcup_{f \in B}\left\{e_{\alpha}-e_{\alpha_{f}}: \alpha \in A_{f} \text { and } \alpha \neq \alpha_{f}\right\}
$$

is linearly independent, of cardinality $\kappa$ and a subset of $\left\langle e_{\beta}: \beta<\lambda\right\rangle^{\perp}$.

In [Sp2], the following forcing to kill Gross spaces was introduced.

Let $F$ be a field. The direct sum $\bigoplus_{n<\omega} F$ of $\omega$ copies of $F$ naturally bears a vector space structure over $F$.

The forcing $P^{F}$ consists of pairs $\langle s, A\rangle$ where

(1) $s=\left\langle s_{0}, \ldots, s_{k}\right\rangle$ is a finite sequence of vectors $s_{i} \in \bigoplus_{n<\omega} F$;

(2) $A \subseteq\left(\bigoplus_{n<\omega} F\right)^{*}$ is a finite set of linear functionals.

The ordering on $P^{F}$ is defined as follows:

$$
\begin{aligned}
& \left\langle s^{\prime}, A^{\prime}\right\rangle \text { extends }\langle s, A\rangle \text { if and only if } s^{\prime} \supseteq s \text { and } A^{\prime} \supseteq A \text { and } \\
& \qquad \forall s_{i}^{\prime} \in \operatorname{ran}\left(s^{\prime}\right) \backslash \operatorname{ran}(s) \forall f \in A f\left(s_{i}^{\prime}\right)=0 .
\end{aligned}
$$

It is easily seen that two conditions with the same first coordinate are compatible. Hence, if $F$ is countable or finite $P^{F}$ has the countable chain condition.

Let $\langle E, \Phi\rangle$ be an inner product space over $F$ and $U$ an arbitrary subspace of infinite dimension. Then forcing with $P^{F}$ introduces in $U$ an infinite set of linearly independent vectors such that every vector in $E$ is orthogonal on all but finitely many of them. Moreover, iterating forcing with $P^{F}$ such that $F$ again and again runs through all finite and countable fields produces a model where Gross spaces of any dimension less than the length of the iteration (which is supposed to be a regular cardinal) do not exist (see [Sp2] for the proofs).

By the Theorem in $\S 2$ above, if $\mathbf{b}=\omega_{1}$ holds a Gross space of dimension $\omega_{1}$ exists over every infinite field. Hence if $F$ is infinite, the forcing $P^{F}$ which kills Gross spaces over $F$ adds a dominating function (see [Sp2]). Here we show that in case $F$ is finite, $P^{F}$ does not destroy unbounded families; more exactly it is almost ${ }^{\omega} \omega$-bounding. Hence, by a preservation theorem in [Sh1] or [Sh2], iterating forcings $P^{F}$ where $F$ runs again and again through all finite fields yields a model where a Gross space in dimension $\omega_{1}$ exists over every infinite field but not over any finite field, provided that we start with a ground model satisfying $\mathbf{b}=\omega_{1}$.

Lemma 2. Assume that $F$ is a finite field and $\tau$ is a $P^{F}$-name for a function in ${ }^{\omega} \omega$. Then there exists $f \in{ }^{\omega} \omega$ in the ground model such that for every $p \in P^{F}$ there are only finitely many $n$ such that $p \Vdash \tau(n)>f(n)$. 
Proof. At first, let us introduce the following notation. If $s=\left\langle s_{0}, \ldots, s_{k}\right\rangle$ is a sequence of vectors in $\bigoplus_{n<\omega} F$ and $\phi$ is a formula in the language of forcing with $P^{F}$ we write $s \Vdash^{*} \phi$ if and only if there exists a finite $A \subset\left(\bigoplus_{n<\omega} F\right)^{*}$ such that $\langle s, A\rangle \Vdash \phi$.

By induction on $i<\omega$ we will define an increasing sequence $\left\langle n_{i}: i<\omega\right\rangle$ such that:

(1) $n_{0}=0$ and

(2) for every $i$, if $s=\left\langle s_{0}, \ldots, s_{k}\right\rangle$ is a sequence of vectors in $\bigoplus_{j<n_{i}} F$ and $f_{0}, \ldots, f_{n_{i}}$ are linear functionals on $\bigoplus_{j<n_{i+1}} F$, then there is a sequence $s^{\prime}$ of vectors in $\bigoplus_{j<n_{i+1}} F$ which extends $s$, such that $\operatorname{ran}\left(s^{\prime}\right) \backslash \operatorname{ran}(s) \subseteq \bigcap_{k \leq n_{i}} \operatorname{ker} f_{k}$ and for some $\sigma \in{ }^{n_{i}} \omega$ we have $s^{\prime} \Vdash^{*} \tau \mid n_{i}=\sigma$.

We have to show that for given $n_{i}$ there exists $n_{i+1}>n_{i}$ such that (2) holds. Assume that this is not true. So we may find $s$ with $\operatorname{ran}(s) \subseteq \bigoplus_{j<n_{i}} F$ such that for infinitely many $j>n_{i}$ there are $f_{0}^{j}, \ldots, f_{n_{i}}^{j} \in\left(\bigoplus_{u<j} F\right)^{*}$ such that there is no $s^{\prime}$ as in the conclusion of (2).

Now every $\left(\bigoplus_{u<j} F\right)^{*}$ is finite. Hence by König's Lemma, there exist $f_{0}, \ldots, f_{n_{i}}$ $\in\left(\bigoplus_{j<\omega} F\right)^{*}$ such that for some $B \in[\omega]^{\omega}$ we have $\forall j \in B \exists j^{\prime}>j \forall k \leq n_{i} f_{k} \uparrow$ $\bigoplus_{u<j} F=f_{k}^{j^{\prime}}\left\lceil\bigoplus_{u<j} F\right.$.

Since $\left\langle s,\left\{f_{0}, \ldots, f_{n_{i}}\right\}\right\rangle$ is a condition in $P^{F}$ there exists a stronger condition $\left\langle s^{\prime}, A^{\prime}\right\rangle$ which decides the first $n_{i}$ values of $\tau$. Choose $j \in B$ so large that $\operatorname{ran}\left(s^{\prime}\right) \subseteq$ $\bigoplus_{u<j} F$. Now for some $j^{\prime}>\max \left\{j, n_{i}\right\}$ we know $\forall k \leq n_{i} f_{k}\left\lceil\bigoplus_{u<j} F=f_{l}^{j^{\prime}} \uparrow\right.$ $\bigoplus_{u<j} F$. But then $s^{\prime}$ satisfies the conclusion of (2) for $s$ and $f_{0}^{j^{\prime}}, \ldots, f_{n_{i}}^{j^{\prime}}$, a contradiction.

Hence we have shown that $\left\langle n_{i}: i<\omega\right\rangle$ can be chosen increasing and satisfying (1) and (2).

Next we define $f: \omega \rightarrow \omega$ as follows. If $n_{i} \leq n<n_{i+1}$, then

$$
\begin{aligned}
& f(n)=\max \{\sigma(n): \text { there exists } s \text { such that } \\
& \qquad \operatorname{ran}(s) \subseteq \bigoplus_{j<n_{i+2}} F \text { and } s \Vdash^{*} \tau\left\lceil n_{i+1}=\sigma\right\} .
\end{aligned}
$$

We claim that $f$ is as desired. So let $\left\langle s,\left\{f_{0}, \ldots, f_{m}\right\}\right\rangle \in P^{F}$ and let $i<\omega$ such that $n_{i}>m$ and $\operatorname{ran}(s) \subseteq \bigoplus_{j<n_{i}} F$. Assume now $n \geq n_{i}$. We will find an extension of $\left\langle s,\left\{f_{0}, \ldots, f_{m}\right\}\right\rangle$ forcing " $f(n) \geq \tau(n)$ ".

Choose $j$ such that $n_{j} \leq n<n_{j+1}$. By construction, there is $s^{\prime}$ extending $s$ such that $\operatorname{ran}\left(s^{\prime}\right) \subseteq \bigoplus_{u<n_{j+2}} F, \operatorname{ran}\left(s^{\prime}\right) \backslash \operatorname{ran}(s) \subseteq \bigcap_{k=1}^{m} \operatorname{ker} f_{k}$ and $s^{\prime} \Vdash^{*} \tau \mid n_{j+1}=\sigma$, for some $\sigma$. Choose a finite $A \subseteq\left(\bigoplus_{u<\omega} F\right)^{*}$ containing $\left\{f_{0}, \ldots, f_{m}\right\}$ such that $\left\langle s^{\prime}, A\right\rangle \Vdash \tau\left\lceil n_{j+1}=\sigma\right.$. Then $\left\langle s^{\prime}, A\right\rangle$ extends $\left\langle s,\left\{f_{0}, \ldots, f_{m}\right\}\right\rangle$ and $\left\langle s^{\prime}, A\right\rangle \Vdash f(n) \geq$ $\tau(n)$.

Remark. It is easy to see that Lemma 2 says much more than that $P^{F}$ is almost ${ }^{\omega} \omega$-bounding (this notion is defined in [Sh2]). In [Sh2] it is proved that this property is preserved under countable support iterations. In the new edition of [Sh1] it is proved that this is true also for finite support iterations. A more accessible proof of this preservation theorem (for the finite support case) can be found in [Go, Example 8.5]. It is not difficult to see that $P^{F}$ adds a Cohen real; hence it is not ${ }^{\omega} \omega$-bounding. 
Corollary. Assume $P$ is a finite or countable support iteration of forcings $P^{F}$ where $F$ is a finite field. If in $V,\left\langle f_{\alpha}: \alpha<\kappa\right\rangle$ is an unbounded family of functions in $\omega^{\omega}$, then $\left\langle f_{\alpha}: \alpha<\kappa\right\rangle$ is unbounded in $V^{P}$.

Proof. Preservation of unboundedness at successor steps of the iteration follows from Lemma 1. Assume $\tau$ is a $P$-name for a function in $\omega^{\omega}$ such that some $p \in P$ forces " $\tau$ bounds $\left\langle f_{\alpha}: \alpha<\kappa\right\rangle$ ". Choose $f \in \omega^{\omega} \cap V$ for $\tau$ as in the Lemma. Since $\left\langle f_{\alpha}: \alpha<\kappa\right\rangle$ is unbounded in $V$ there exists $\alpha$ such that for infinitely many $n$ we have $f(n)<f_{\alpha}(n)$. Find $p^{\prime} \in P$ extending $p$ and $n_{0}$ such that $p^{\prime} \Vdash \forall n \geq n_{0} \tau(n)>f_{\alpha}(n)$. But then for infinitely many $n$ we would have $p^{\prime} \Vdash \tau(n)>f(n)$, a contradiction.

The limit steps are handled by a general preservation theorem due to the first author (see [Sh2] or [Sh1, new ed., VI§3]).

From the Corollary, the Theorem in $\S 2$ and the results about forcing with $P^{F}$ 's in [Sp2] we obtain the following theorem.

Theorem 1. Assume that the ground model $V$ satisfies $\mathbf{b}=\omega_{1}$. Let

$$
P=\lim \left\langle P_{\alpha}, Q_{\alpha}: \alpha<\kappa\right\rangle
$$

be a finite or countable support iteration of length $\kappa$ where $\kappa>\omega_{1}$ is regular such that

(1) each $Q_{\alpha}$ is $P^{F}$ defined in $V^{P_{\alpha}}$ where $F$ is a finite field, and

(2) for each finite field $F$, cofinally many times we have $Q_{\alpha}=P^{F}$.

Then in $V^{P}$, there exists a Gross space of dimension $\omega_{1}$ over every infinite field but there is no Gross space of dimension $\kappa$ over any finite field.

Another way to get a model for the conclusion of the Theorem above is shown by the following Lemma combined with Theorem $3, \S 1$, and the Theorem in $\S 2$.

Lemma 3. Assume that $\langle E, \Phi\rangle$ is a symmetric bilinear space over a finite field and the dimension of $E$ has uncountable cofinality. If $\mathbf{s}\rangle \operatorname{dim} E$ then $\langle E, \Phi\rangle$ is not Gross.

Proof. Let $\operatorname{dim} E=\kappa$. In $E$, choose a basis $\left\langle e_{\alpha}: \alpha<\kappa\right\rangle$ and let $\left\langle y_{n}: n<\omega\right\rangle$ span a subspace. For every $\alpha<\kappa$ let

$$
f_{\alpha}: \omega \rightarrow F, n \mapsto \Phi\left(e_{\alpha}, y_{n}\right)
$$

where $F$ is the base field. Let $\mathcal{A}=\left\langle f_{\alpha}^{-1}\{a\}: \alpha<\kappa, a \in F\right\rangle$. Since $\mathbf{s}>\kappa, \mathcal{A}$ is not a splitting family. Hence there is an infinite $A \subseteq \omega$ such that for all $B \in \operatorname{ran} \mathcal{A}$, either $A \subseteq^{*} B$ or $A \cap B$ is finite. For any $\alpha<\kappa,\left\langle f_{\alpha}^{-1}\{a\}, a \in F\right\rangle$ is a finite partition of $\omega$. So there exist $a_{\alpha}$ and $n_{\alpha}$ such that $A \backslash n_{\alpha} \subseteq f_{\alpha}^{-1}\left\{a_{\alpha}\right\}$. By $\operatorname{cf}(\kappa)>\omega$ there exist $a, n$ and $X \in[\kappa]^{\kappa}$ such that for every $\alpha \in X$ we have $a_{\alpha}=a$ and $n_{\alpha}=n$. Now it is easy to see that every vector $e_{\alpha}-e_{\beta}$ with $\alpha, \beta \in X$ is in the orthogonal complement of $\operatorname{span}\left\langle y_{m}, m \in A \backslash n\right\rangle$.

The model from [B/Sh] where simultaneously simple $P_{\aleph_{1}}$ - and $P_{\aleph_{2}}$-points exist shows that a stronger statement than the conclusion of Theorem 1 is true. A $P_{\kappa^{-}}$ point, where $\kappa$ is a regular uncountable cardinal, is a filter on $\omega$ which is generated by a tower (i.e. a $\subseteq^{*}$-decreasing family in $[\omega]^{\omega}$ ) of length $\kappa$.

Theorem 2. It is consistent that a Gross space of dimension $\omega_{1}$ exists over every infinite field but no Gross space exists over any finite field in any dimension. 
Proof. In the model of [B/Sh] where simple $P_{\aleph_{1}-}$ and $P_{\aleph_{2}}$-points exist, $\mathbf{b}=\omega_{1}$ holds. Hence by the Theorem in $\S 2$, a Gross space of dimension $\aleph_{1}$ exists over any infinite field. Using a similar argument as in the proof of Lemma 3, one shows that a $P_{\aleph_{1}-}, P_{\aleph_{2}}$-point rules out the existence of a Gross space over any finite field in dimension $\aleph_{1}, \aleph_{2}$ respectively. But in that model $\mathbf{c}=\omega_{2}$. Hence by Lemma 1 we are done.

Question. Does there exist a ZFC-model where there exists no Gross space over any finite or countable field in any dimension?

In view of the Theorem in $\S 2$, a natural question to ask is whether the assumption $\mathbf{s}=\omega_{1}$ is strong enough to construct a Gross space of dimension $\aleph_{1}$ over a finite field. The answer is "no".

Theorem 3. It is consistent that $\mathbf{s}=\omega_{1}$ holds and there exists no Gross space in dimension $\omega_{1}$ over any finite field.

Proof. Since every finite or countable field can be coded by a real, it is not difficult to see that the forcing notion $P^{F}$, where $F$ is finite or countable, is Souslin in the sense of $[\mathrm{J} / \mathrm{Sh}]$; i.e., the set of conditions can be viewed as an analytic set of reals, whereas the ordering and the incompatibility relation are analytic subsets of the plane. In $[\mathrm{J} / \mathrm{Sh}]$ it is proved that if we start with a model satisfying $\mathrm{CH}$, then in any finite support extension of Souslin forcing notions having the countable chain condition, the reals of the ground model remain a splitting family. Hence in $V^{P}$, where $P$ is the finite support iteration of Theorem 1 and $V$ satisfies $\mathrm{CH}$, $\mathbf{s}=\omega_{1}$ holds and there are no Gross spaces in any dimension $<\mathbf{c}$ of uncountable cofinality.

Is there another cardinal invariant such that its being $\aleph_{1}$ implies that Gross spaces over finite fields exist? The largest of the "classical" cardinal invariants is d. The model in [Sh3, §2] shows that even $\mathbf{d}=\omega_{1}$ does not suffice for our purpose. In that model, if $\langle E, \Phi\rangle$ is a quadratic space spanned by a basis $\left\langle e_{\alpha}: \alpha<\omega_{1}\right\rangle$ over some finite field $F$, then the following holds:

Fact. If $\left\langle u_{n}: n\langle\omega\rangle\right.$ is a sequence of pairwise disjoint subsets of $\omega$ such that every $u_{n}$ has size $|F|^{n}+1$, then we can find $\left\langle H_{n}: n<\omega\right\rangle$, where each $H_{n}$ is a family of at most $n$ functions $u_{n} \rightarrow F$, such that

$$
\forall \alpha<\omega_{1} \exists n_{\alpha}<\omega \forall n \geq n_{\alpha} \exists h \in H_{n} \forall m \in u_{n}\left[\Phi\left(e_{\alpha}, e_{m}\right)=h(m)\right] .
$$

It is not difficult to see that this implies that $\langle E, \Phi\rangle$ is not Gross (solves many systems of homogeneous equations).

Next we will show that if there is a family of $\aleph_{1}$, many meagre sets of reals which are cofinal with respect to inclusion in the set of all meagre sets, then a Gross space of dimension $\aleph_{1}$ exists over every finite field. We work in $2^{\omega}$ considered as the topological product of the discrete space $2=\{0,1\}$. Denote by $\mathcal{M}$ the set of all meagre sets in $2^{\omega}$. Now $\operatorname{cof}(\mathcal{M})$ is the cardinal invariant defined as follows:

$$
\begin{aligned}
\operatorname{cof}(\mathcal{M})=\min \{|\mathcal{F}|: & \mathcal{F} \text { is a family of meagre sets } \\
& \text { such that } \forall A \in \mathcal{M} \exists B \in \operatorname{ran} \mathcal{F} A \subseteq B\} .
\end{aligned}
$$

Theorem 4. Assume $\operatorname{cof}(\mathcal{M})=\omega_{1}$. A Gross space of dimension $\aleph_{1}$ exists over every finite field. 
Proof. Using the assumption it is standard work to construct a family $\left\langle r_{\alpha}: \alpha<\omega_{1}\right\rangle$ of reals such that, if $A \subseteq\left(2^{\omega}\right)^{n}$ is a meagre set for some $n$, then $\exists \alpha<\omega_{1} \forall \alpha<\alpha_{1}<$ $\ldots<\alpha_{n}\left\langle r_{\alpha_{1}}, \ldots, r_{\alpha_{n}}\right\rangle \notin A$.

By induction on $\alpha<\omega_{1}$ choose one-to-one functions $h_{\alpha}: \alpha \rightarrow \omega$ such that $\omega \backslash \operatorname{ran}\left(h_{\alpha}\right)$ is infinite and for every $\beta<\alpha$ the set $\left\{\gamma<\beta: h_{\beta}(\gamma) \neq h_{\alpha}(\gamma)\right\}$ is finite. (This is a well-known construction of an Aronszajn tree due to Todorcevic.)

Now let $F$ be a field and $E$ a vector space over $F$ of dimension $\aleph_{1}$, spanned by a basis $\left\langle e_{\alpha}: \alpha<\omega_{1}\right\rangle$. Define a symmetric bilinear form $\Phi$ on $E$ as follows: For $\alpha<\beta<\omega_{1}$ set

$$
\Phi\left(e_{\alpha}, e_{\beta}\right)=r_{\beta}\left(h_{\beta}(\alpha)\right) .
$$

We claim that $\langle E, \Phi\rangle$ is Gross. If this is not true there are families of vectors $\left\langle y_{k}: k<\omega\right\rangle$ and $\left\langle z_{\iota}: \iota<\omega_{1}\right\rangle$ such that $\Phi\left(y_{k}, z_{\iota}\right)=0$ always, $\left\langle\operatorname{dom}\left(y_{k}\right): k<\omega\right\rangle$ and $\left\langle\operatorname{dom}\left(z_{\iota}\right): \iota<\omega_{1}\right\rangle$ are families of pairwise disjoint sets, and the sets of the latter have all the same cardinality and are disjoint from some $\alpha^{*}<\omega_{1}$ such that $\operatorname{span}\left\langle y_{k}: k<\omega_{1}\right\rangle \subseteq \operatorname{span}\left\langle e_{\alpha}: \alpha<\alpha^{*}\right\rangle$. Let

$$
y_{k}=\sum_{l=1}^{m_{k}} b_{k l} e_{\alpha(k, l)}, \quad z_{\iota}=\sum_{l=1}^{n} c_{l} e_{\beta(\iota, l)}
$$

such that each $m_{k}>0$ and $n>0$, each $b_{k l}, c_{l} \neq 0$, and $\beta(\iota, 1)<\ldots<\beta(\iota, n)$ always.

Define $A \subseteq\left(2^{\omega}\right)^{n}$ as follows:

$$
A=\left\{\left\langle r_{1}, \ldots, r_{n}\right\rangle:\left\{k: \sum_{1 \leq l \leq m_{k}} \sum_{1 \leq l^{\prime} \leq n} b_{k l} c_{l^{\prime}} r_{l^{\prime}}\left(h_{\alpha^{*}}(\alpha(k, l))\right) \neq 0\right\} \text { is finite }\right\} .
$$

It is not difficult to see that $A$ is meagre. Hence by construction we may choose $\iota$ so large that $\left\langle r_{\beta(\iota, 1)}, \ldots, r_{\beta(\iota, n)}\right\rangle \notin A$. Consequently, by the choice of $h_{\alpha}$ we may find $k$ such that

$$
\begin{aligned}
\Phi\left(y_{k}, z_{\iota}\right) & =\sum_{1 \leq l \leq m_{k}} \sum_{1 \leq l^{\prime} \leq n} b_{k l} c_{l^{\prime}} r_{\beta\left(\iota, l^{\prime}\right)}\left(h_{\beta\left(\iota, l^{\prime}\right)}(\alpha(k, l))\right) \\
& =\sum_{1 \leq l \leq m_{k}} \sum_{1 \leq l^{\prime} \leq n} b_{k l} c_{l^{\prime}} r_{\beta\left(\iota, l^{\prime}\right)}\left(h_{\alpha^{*}}(\alpha(k, l))\right) \neq 0 .
\end{aligned}
$$

This is a contradiction.

Remark. Theorem 4 is true if the assumption $\operatorname{cof}(\mathcal{M})=\omega_{1}$ is replaced by $\operatorname{cof}(\mathcal{N})=$ $\omega_{1}$ where $\mathcal{N}$ denotes the set of all Lebesgue measure zero sets of reals. The same proof works if we replace "meagre" by "measure zero". The proof also works for arbitrary (not necessarily finite) fields. All this is not astonishing since Cichoń's diagram (see $[\mathrm{F}]$ ) tells us that $\mathbf{b} \leq \mathbf{d} \leq \operatorname{cof}(\mathcal{M}) \leq \operatorname{cof}(\mathcal{N})$.

\section{Generalized Gross Spaces exist in ZFC}

Here we consider a natural generalization of the Gross property and will obtain results in $\mathrm{ZFC}$.

Let $F$ be a field of arbitrary cardinality and $\langle E, \Phi\rangle$ a symmetric bilinear space over $F$, and let $\lambda$ be an infinite cardinal. We say that $\langle E, \Phi\rangle$ is a $\lambda$-Gross space if it has the following property:

$(* * *)$ for all subspaces $U \subseteq E$ of dimension $\geq \lambda$ : $\operatorname{dim} U^{\perp}<\operatorname{dim} E$. 
So a Gross space is an $\omega$-Gross space. By Lemma 1 in $\S 3,|F|^{\lambda}$ is an upper bound for the dimension of a $\lambda$-Gross space over $F$.

By results from $[\mathrm{B} / \mathrm{G}]$ and $[\mathrm{B} / \mathrm{Sp}]$ the existence of Gross spaces of dimension $\aleph_{1}$ over countable or finite fields is independent of ZFC. We will prove here that in ZFC an $\omega_{1}$-Gross space of dimension $\aleph_{1}$ can be constructed over any countably infinite field.

We need the following fancy lemma.

Lemma 1. Let $F$ be an infinite field. There exists a sequence $\left\langle a_{n}: n<\omega\right\rangle$ of elements of $F$ such that, whenever $\left\langle k_{i j}: 1 \leq i, j \leq m\right\rangle$ is a finite sequence of pairwise distinct integers, then

$$
\left|\begin{array}{ccc}
a_{k_{11}} & \ldots & a_{k_{1 m}} \\
\vdots & & \vdots \\
a_{k_{m 1}} & \ldots & a_{k_{m m}}
\end{array}\right| \neq 0 .
$$

Proof. This is accomplished by a fusion argument. Start with a sequence $\left\langle a_{n}^{1}: n<\right.$ $\omega\rangle$ of pairwise distinct scalars in $F \backslash\{0\}$. Then clearly every subsequence satisfies the conclusion of the Lemma for $m=1$.

Now assume $\left\langle a_{n}^{m}: n<\omega\right\rangle$ has been constructed such that the conclusion of the Lemma holds for any $1 \leq m^{\prime} \leq m$. Let $n_{i}=i$ for any $i \leq(m+1)^{2}-1$. Assume $n_{0}, \ldots, n_{l}$ have been chosen.

Let $\left\langle i_{0}, j_{0}\right\rangle \in\{1, \ldots, m+1\}^{2}$ and let $\left\langle k_{i j}:\langle i, j\rangle \in\{1, \ldots, m+1\}^{2} \backslash\left\langle i_{0}, j_{0}\right\rangle\right\rangle$ be a sequence of pairwise distinct integers in $\left\{n_{0}, \ldots, n_{l}\right\}$. By the induction hypothesis, the equation

$$
i_{0} \rightarrow\left|\begin{array}{ccc}
j_{0} & \\
a_{k_{11}}^{m} & \downarrow & a_{k_{1 m+1}}^{m} \\
\vdots & X & \vdots \\
a_{k_{m+11}}^{m} & \cdots & a_{k_{m+1 m+1}}^{m}
\end{array}\right|=0
$$

in one variable $X$ which has coordinates $\left\langle i_{0} j_{0}\right\rangle$ has exactly one solution. (Expand the determinant by the $i_{0}$ th row. Then the cofactors are all nonzero.)

Now choose $n_{l+1}>n_{l}$ minimal such that for any $n \geq n_{l+1}, a_{n}^{m}$ is distinct from all the finitely many solutions of the equation above obtained by running through all possible $\left\langle i_{0}, j_{0}\right\rangle$ 's and $\left\langle k_{i j}:\langle i, j\rangle \in\{1, \ldots m+1\}^{2} \backslash\left\langle i_{0} j_{0}\right\rangle\right\rangle$ 's.

Now define $a_{l}^{m+1}=a_{n_{l}}^{m}, l<\omega$.

Finally define

$$
a_{n}=a_{n}^{m} \text { iff } n \in\left\{m^{2}-1, \ldots,(m+1)^{2}-2\right\} .
$$

It is easily checked that $\left\langle a_{n}: n<\omega\right\rangle$ is as desired.

For uncountable fields an analog of Lemma 1 is true which is more obvious. This is essentially [B/Sp, Lemma $2, \S 4]$.

Lemma 2. Let $F$ be a field of uncountable cardinality $\lambda$. There exists a sequence $\left\langle a_{\nu}: \nu<\lambda\right\rangle$ of elements of $F$ such that, whenever $\left\langle\nu_{i j}: 1 \leq i, j \leq m\right\rangle$ is a finite 
sequence of pairwise distinct ordinals $<\lambda$, then

$$
\left|\begin{array}{ccc}
a_{\nu_{11}} & \ldots & a_{\nu_{1 m}} \\
\vdots & & \vdots \\
a_{\nu_{m 1}} & \ldots & a_{\nu_{m m}}
\end{array}\right| \neq 0 .
$$

Proof. Let $\left\langle a_{\nu}: \nu<\lambda\right\rangle$ be a one-to-one enumeration of a transcendence base of $F$ over its prime field. Prove by the induction on the matrix size $m$ that the conclusion is satisfied. Expand the determinant by, say, the first row. Then by induction hypothesis the cofactors are all nonzero, and the transcendentals of the first row do not occur in them.

Theorem 1. An $\omega_{1}$-Gross space of dimension $\aleph_{1}$ exists over every countably infinite field.

Proof. Let $F$ be a countably infinite field. Choose scalars $\left\langle a_{n}: n<\omega\right\rangle$ in $F$ as in Lemma 1. Let $\left\langle A_{\alpha}: \alpha<\omega_{1}\right\rangle$ be an almost disjoint family of subsets of $\operatorname{ran}\left\langle a_{n}: n<\omega\right\rangle$. For each $\alpha<\omega_{1}$ choose a one-to-one function $f_{\alpha}: \alpha \rightarrow A_{\alpha}$. Let $E$ be a vector space over $F$ of dimension $\aleph_{1}$ and let $\left\langle e_{\alpha}: \alpha<\omega_{1}\right\rangle$ be a basis of $E$.

On $E$, define a symmetric bilinear form as follows: For $\alpha<\beta<\omega_{1}$ define

$$
\Phi\left(e_{\alpha}, e_{\beta}\right)=f_{\beta}(\alpha)
$$

$\Phi\left(e_{\alpha}, e_{\alpha}\right)$ may be defined arbitrarily.

We claim that $\langle E, \Phi\rangle$ is as desired. Using a $\Delta$-system argument and the bilinearity of $\Phi$, it is not difficult to see that it is enough to prove the following.

$(\star)$ Assume that $U$ is a subspace spanned by a basis $\left\langle y_{k}: k<\omega\right\rangle$ such that the sets in $\left\langle\operatorname{supp}\left(y_{k}\right): k<\omega\right\rangle$ are pairwise disjoint and of the same cardinality, then $\operatorname{dim} U^{\perp}<\omega_{1}$.

By way of contradiction assume that $U$ and $\left\langle y_{k}: k<\omega\right\rangle$ are as in $(\star)$ but $U^{\perp}$ contains $\omega_{1}$ many linearly independent vectors $\left\langle z_{\iota}: \iota<\omega_{1}\right\rangle$.

Let

$$
y_{k}=\sum_{l=1}^{m} b_{k l} e_{\alpha(k, l)} .
$$

We may assume that each $z_{\iota}$ has the same nonzero coefficients in its representation, say

$$
z_{\iota}=\sum_{l=1}^{n} c_{l} e_{\beta(\iota, l)}
$$

and for any $\iota<\nu<\omega_{1}$ the $\operatorname{sets} \operatorname{supp}\left(z_{\iota}\right)$ and $\operatorname{supp}\left(z_{\nu}\right)$ are disjoint and $\operatorname{supp}\left(z_{\iota}\right)$ is disjoint from $\sup \bigcup_{k<\omega} \operatorname{supp}\left(y_{k}\right)$.

We claim that not even the first $m \cdot n$ many $z_{\iota}$ 's are in $U^{\perp}$. In order to see this, let us compute

$$
\begin{gathered}
\Phi\left(z_{\iota}, y_{k}\right)=c_{1} b_{k 1} f_{\beta(\iota, 1)}(\alpha(k, 1))+\ldots+c_{1} b_{k m} f_{\beta(\iota, 1)}(\alpha(k, m)) \\
\vdots \\
+c_{n} b_{k 1} f_{\beta(\iota, n)}(\alpha(k, 1))+\ldots+c_{n} b_{k m} f_{\beta(\iota, n)}(\alpha(k, m)) .
\end{gathered}
$$

Since the sets $A_{\alpha}$ are almost disjoint, the sets $\operatorname{supp}\left(y_{k}\right)=\{\alpha(k, 1), \ldots, \alpha(k, m)\}$ are pairwise disjoint, the functions $f_{\alpha}$ are one-to-one and the scalars $\left\langle a_{n}: n<\omega\right\rangle$ 
are chosen satisfying the conclusion of Lemma 1, there is $k<\omega$ such that for $\iota<m \cdot n$ the sets

$$
\left\{f_{\beta(\iota, i)}(\alpha(k, j)): 1 \leq i \leq n, 1 \leq j \leq m\right\}
$$

are pairwise disjoint, contain $m \cdot n$ elements and

$$
\left|\begin{array}{ccc}
f_{\beta(0,1)}(\alpha(k, 1)) & \ldots & f_{\beta(0, n)}(\alpha(k, m)) \\
\vdots & & \vdots \\
f_{\beta(m n-1,1)}(\alpha(k, 1)) & \ldots & f_{\beta(m n-1, n)}(\alpha(k, m))
\end{array}\right| \neq 0 .
$$

But then $y_{k}$ is not orthogonal on every $z_{\iota}, \iota<m \cdot n$, since otherwise the vector

$$
\left\langle c_{1} b_{k 1}, \ldots, c_{n} b_{k m}\right\rangle \in F^{m n}
$$

would be a nontrivial solution of the system of homogeneous equations $A x=0$ where $A$ is the $m n \times m n$-matrix in (1).

The upper bound for the dimension of an $\omega_{1}$-Gross space over a countable field is $\omega^{\omega_{1}}=2^{\omega_{1}}$, by Lemma $1, \S 3$. Hence, the largest dimension in which such a space conceivably can be constructed in ZFC is $\omega_{2}$. Theorem 6 below will show that in fact such a construction is possible.

For the following question we have no answer.

Question. Does there exist an $\omega_{1}$-Gross space of uncountable dimension over any finite field?

Next we will show that this question has a positive answer for regular cardinals larger than $\omega_{1}$. For this, recent work of the first author on colouring pairs of ordinals will be applied. Let $\lambda, \mu, \kappa, \theta$ be cardinals such that $\lambda$ is infinite and $\lambda \geq \mu \geq \kappa+\theta$. Let $\operatorname{Pr}_{0}(\lambda, \mu, \kappa, \theta)$ be the following statement.

$\operatorname{Pr}_{0}(\lambda, \mu, \kappa, \theta)$ : There exists a function $c:[\lambda]^{2} \rightarrow \kappa$ such that, if $\xi<\theta$ and for $\nu<\mu,\left\langle\alpha_{\nu, \zeta}: \zeta<\xi\right\rangle$ is a strictly increasing sequence of ordinals $<\lambda$, the $\alpha_{\nu, \zeta}$ distinct, and $h: \xi \times \xi \rightarrow \kappa$, then there are $\nu_{1}<\nu_{2}<\mu$ such that for all $\zeta_{1}, \zeta_{2}<\xi$ we have $c\left\{\alpha_{\nu_{1}, \zeta_{1}}, \alpha_{\nu_{2}, \zeta_{2}}\right\}=h\left\langle\zeta_{1}, \zeta_{2}\right\rangle$.

The following theorem, of the first author, states that $\operatorname{Pr}_{0}\left(\lambda^{+}, \lambda^{+}, \lambda^{+}, \omega\right)$. holds for all regular uncountable cardinals. For regular $\lambda>\omega_{1}$ this is proved in [Sh4, Corollary (a), Section 4, p.100]; see Definition 1, p. 95. For $\lambda=\omega_{1}$ this was proved in [Sh7, Theorem 1.1].

Theorem 2. $\operatorname{Pr}_{0}\left(\lambda^{+}, \lambda^{+}, \lambda^{+}, \omega\right)$ and hence $\operatorname{Pr}_{0}\left(\lambda^{+}, \lambda^{+}, 2, \omega\right)$ holds for every regular uncountable cardinal $\lambda$.

For more on Theorem 2 (for $\lambda>\omega_{1}$ ) see also [Sh5]. Theorem 2 appears (when we specify $\theta=\omega)$ in [Sh5, III, 4.8(1), p.177]. Moreover, 4.8(2) gives similar results for inaccessibles with stationary subsets not reflecting in inaccessibles.

Theorem 3. Let $\lambda$ be a regular uncountable cardinal. $A \lambda^{+}$-Gross space of dimension $\lambda^{+}$exists over any field.

Proof. Let $F$ be an arbitrary field of size at most $\lambda$ (for larger fields see Theorem 4 below) and $E$ a vector space over $F$ of dimension $\lambda^{+}$. Choose a basis $\left\langle e_{\alpha}\right.$ : $\left.\alpha<\lambda^{+}\right\rangle$. We define a symmetric bilinear form on $E$ using the colouring given by $\operatorname{Pr}_{0}\left(\lambda^{+}, \lambda^{+}, 2, \omega\right)$. For $\{\alpha, \beta\} \in\left[\lambda^{+}\right]^{2}$ we set

$$
\Phi\left(e_{\alpha}, e_{\beta}\right)=c\{\alpha, \beta\} .
$$


The angles $\Phi\left(e_{\alpha}, e_{\alpha}\right)$ may be defined arbitrarily.

Assume that $\langle E, \Phi\rangle$ is not $\lambda^{+}$-Gross. So we may find families $\left\langle y_{\nu}: \nu\left\langle\lambda^{+}\right\rangle\right.$ and $\left\langle z_{\nu}: \nu<\lambda^{+}\right\rangle$of linearly independent vectors such that $\Phi\left(y_{\nu}, z_{\nu^{\prime}}\right)=0$ always. Without loss of generality we may assume that $\left\langle\operatorname{dom}\left(y_{\nu}\right): \nu\left\langle\lambda^{+}\right\rangle\right.$and $\left\langle\operatorname{dom}\left(z_{\nu}\right)\right.$ : $\left.\nu<\lambda^{+}\right\rangle$are families of pairwise disjoint sets, the sets in the first one all of size $n$ and those in the latter all of size $m$, and if $\nu<\nu^{\prime}$ and $\alpha \in \operatorname{dom}\left(y_{\nu}\right), \beta \in \operatorname{dom}\left(z_{\nu}\right)$, $\gamma \in \operatorname{dom}\left(y_{\nu^{\prime}}\right)$ then $\alpha<\beta<\gamma$. Furthermore we may assume that each $y_{\nu}$ and each $z_{\nu}$ has the same nonzero coefficients in its representation, say

$$
y_{\nu}=\sum_{l<n} b_{l} e_{\beta(\nu, l)}, \quad z_{\nu}=\sum_{l<m} c_{l} e_{\gamma(\nu, l)} .
$$

We assume that $\beta(\nu, l)$ and $\gamma(\nu, l)$ increase with $l$. Let $\left\langle\alpha_{\nu, i}: i<n+m\right\rangle$ be the increasing enumeration of $\operatorname{dom}\left(y_{\nu}\right) \cup \operatorname{dom}\left(z_{\nu}\right)$.

It is easy to define $h:(n+m) \times(n+m) \rightarrow 2$ such that

$$
\sum_{l<n} \sum_{l^{\prime}<m} b_{l} c_{l^{\prime}} h\left\langle l, n+l^{\prime}\right\rangle \neq 0 .
$$

But now by $\operatorname{Pr}_{0}\left(\lambda^{+}, \lambda^{+}, 2, \omega\right)$ we may find $\nu_{1}<\nu_{2}<\lambda^{+}$such that for all $i, j<n+m$ we have $c\left\{\alpha_{\nu_{1}, i}, \alpha_{\nu_{2}, j}\right\}=h\langle i, j\rangle$. We conclude $\Phi\left(y_{\nu_{1}}, z_{\nu_{2}}\right) \neq 0$, a contradiction.

For uncountable fields of cardinality equal to the dimension of the space a much stronger version of Theorems 1 and 3 was proved in [G/O].

Theorem 4 [G/O, Theorem $1, \S 1]$. Let $F$ be a field of uncountable cardinality $\lambda$. $A$ strong Gross space of dimension $\lambda$ over $F$ exists.

Here a strong Gross space is a space $\langle E, \Phi\rangle$ satisfying the following property:

$(* *)$ for all subspaces $U \subseteq E$ of infinite dimension: $\operatorname{dim} U^{\perp} \leq \aleph_{0}$. For more on strong Gross spaces see [Sp4].

Constructing a Gross space gets more difficult, the larger its dimension is compared with the size of its base field. For $\lambda=\omega$, the existence of a $\lambda$-Gross space of dimension $\lambda^{+}$over some field of size $\lambda$ is independent of ZFC. The next theorem shows that if $\lambda$ is uncountable, then such a space can be constructed in ZFC. If $\operatorname{cf}(\lambda)>\omega$, a similar construction as in Theorem 1 is possible. If $\operatorname{cf}(\lambda)=\omega$, the almost disjoint sets used for the construction must be chosen more carefully. For this we will need the following result from the first author's work on cardinal arithmetic. It is obtained from [Sh6, 6.2] with the ideal of bounded subsets of $\kappa$ and the $f_{\alpha}$ 's coming from [Sh5, II, 1.5, p.50].

Lemma $3[\mathrm{Sh} 6,6.2]$. Assume that $\lambda$ is a singular cardinal and $\operatorname{cf}(\lambda)=\kappa$. There exists an increasing sequence $\left\langle\lambda_{\iota}: \iota<\kappa\right\rangle$ of regular cardinals $\lambda_{\iota}>\kappa$ with limit $\lambda$ and a family $\left\langle f_{\alpha}: \alpha<\lambda^{+}\right\rangle$of functions in $\prod_{\iota<\kappa} \lambda_{\iota}$ such that for all $\alpha<\beta<\lambda^{+}$:

(1) $f_{\alpha}<f_{\beta}$ modulo bounded subsets of $\kappa$, and

(2) if $\left\langle u_{\zeta}: \zeta<\lambda^{+}\right\rangle$is a sequence of pairwise disjoint nonempty subsets of $\lambda^{+}$, each $u_{\zeta}$ of size $<\kappa$ and $\mu<\lambda$ is a cardinal, then there exists $B \subseteq \lambda^{+}$of size $\mu$ and $\iota^{*}<\kappa$ such that for all $\zeta, \xi \in B$ and $\iota<\kappa$ :

(i) if $\zeta<\xi$, then $\sup u_{\zeta}<\min u_{\xi}$, and

(ii) if $\alpha, \beta \in \bigcup_{\zeta \in B} u_{\zeta}, \alpha<\beta$ and $\iota^{*} \leq \iota<\kappa$, then $f_{\alpha}(\iota)<f_{\beta}(\iota)$.

Theorem 5. Let $\lambda$ be an uncountable cardinal and $F$ a field of size $\lambda$. Then a $\lambda$-Gross space over $F$ of dimension $\lambda^{+}$exists. 
Proof. Let $F$ be a field of cardinality $\lambda$. Let $\left\langle a_{\nu}: \nu<\lambda\right\rangle$ be a transcendence base of $F$ over its prime field $F_{0}$. Hence, $F$ is an algebraic extension of $F_{0}\left(\left\langle a_{\nu}: \nu<\lambda\right\rangle\right)$. Let $E$ be a $F$-vector space of dimension $\lambda^{+}$, spanned by a basis $\left\langle e_{\alpha}: \alpha<\lambda^{+}\right\rangle$.

Case 1. Assume $\operatorname{cf}(\lambda)>\omega$. Choose $\left\langle A_{\alpha}: \alpha<\lambda^{+}\right\rangle$, a family of almost disjoint subsets of $\lambda$, i.e. each $A_{\alpha}$ has size $\lambda$ and if $\alpha, \beta$ are distinct, then $\left|A_{\alpha} \cap A_{\beta}\right|<\lambda$ (see [J, p.252]). For each $\alpha<\lambda^{+}$choose a one-to-one function $g_{\alpha}: \alpha \rightarrow A_{\alpha}$.

On $E$, define a symmetric bilinear form as follows: for $\alpha<\beta<\lambda^{+}$let

$$
\Phi\left(e_{\alpha}, e_{\beta}\right)=a_{\nu} \text { if and only if } g_{\beta}(\alpha)=\nu .
$$

The angles $\Phi\left(e_{\alpha}, e_{\alpha}\right)$ may be defined arbitrarily.

We claim that $\langle E, \Phi\rangle$ is a $\lambda$-Gross space. Since $\operatorname{cf}(\lambda)>\omega$, in every subspace $U \subseteq E$ of dimension $\lambda$ we may find $\lambda$ many vectors such that the supports of each of them has the same size. We also may assume that these supports are pairwise disjoint (see Case 2). Hence it is enough to prove the following.

$(\star \star)$ Assume that $U$ is a subspace spanned by a basis $\left\langle y_{\nu}: \nu<\lambda\right\rangle$ such that the sets in $\left\langle\operatorname{supp}\left(y_{\nu}\right): \nu<\lambda\right\rangle$ are pairwise disjoint and of the same cardinality. Then $\operatorname{dim} U^{\perp}<\operatorname{dim} E$.

The proof of this is completely analogous to that of $(\star)$ in the proof of Theorem 1 , if we use Lemma 2 instead of Lemma 1.

Case 2. Assume $\operatorname{cf}(\lambda)=\omega$. In this case, we have to choose the almost disjoint sets $\left\langle A_{\alpha}: \alpha<\lambda^{+}\right\rangle$more carefully. Let $\left\langle\lambda_{n}: n<\omega\right\rangle$ and $\left\langle f_{\alpha}: \alpha<\lambda^{+}\right\rangle$be as in Lemma 3 where $\kappa=\omega$.

Let $\left\langle\mu_{n}: n<\omega\right\rangle$ be increasing, continuous and with limit $\lambda$, such that $\mu_{n}<\lambda_{n}$ for every $n$. For each $\alpha<\lambda^{+}$define

$$
A_{\alpha}=\bigcup_{n<\omega}\left[\mu_{n}+\mu_{n} \cdot f_{\alpha}(n), \mu_{n}+\mu_{n} \cdot\left(f_{\alpha}(n)+1\right)\right.
$$

and let $g_{\alpha}: \alpha \rightarrow A_{\alpha}$ be one-to-one and onto.

Now let $E$ be a vector space over $F$ of dimension $\lambda^{+}$, spanned by a basis $\left\langle e_{\alpha}\right.$ : $\left.\alpha<\lambda^{+}\right\rangle$. On $E$, define a symmetric bilinear form as follows: if $\alpha<\beta<\lambda^{+}$, let

$$
\Phi\left(e_{\alpha}, e_{\beta}\right)=a_{g_{\beta}(\alpha)} .
$$

The angles $\Phi\left(e_{\alpha}, e_{\alpha}\right)$ may be defined arbitrarily.

We claim that $\langle E, \Phi\rangle$ is a $\lambda$-Gross space. Assume that this is not true. So there is a subspace $U$, spanned by a basis $\left\langle y_{\nu}: \nu<\lambda\right\rangle$, such that $U^{\perp}$ contains a family $\left\langle z_{\zeta}: \zeta\left\langle\lambda^{+}\right\rangle\right.$of linearly independent vectors. Without loss of generality we may and do assume that $\left\langle\operatorname{supp}\left(y_{\nu}\right): \nu\langle\lambda\rangle\right.$ is a family of pairwise disjoint sets. This may be seen as follows: for each $n<\omega$ there exists $Y_{n} \subseteq\left[\lambda_{n}, \lambda_{n}^{+}\right)$of size $\lambda_{n}^{+}$such that $\left\langle\operatorname{supp}\left(y_{\nu}\right): \nu \in Y_{n}\right\rangle$ is a $\Delta$-system. By forming linear combinations, we find $X_{n} \subseteq \operatorname{span}\left\langle y_{\nu}: \nu \in Y_{n}\right\rangle$ such that $\left\langle\operatorname{supp}(x): x \in X_{n}\right\rangle$ is a pairwise disjoint family. By cutting off from each $X_{n}$ at most $\sum_{n^{\prime}<n} \lambda_{n^{\prime}}$ many elements, we obtain a family as desired.

Let

$$
y_{\nu}=\sum_{l=1}^{m_{\nu}} b_{\nu l} e_{\alpha(\nu, l)}
$$

where each $b_{\nu l}$ is nonzero. Choose $\alpha^{*}<\lambda^{+}$such that $\forall \nu<\lambda \forall 1 \leq l \leq m_{\nu}$, $\alpha(\nu, l)<\alpha^{*}$. 
Without loss of generality we may assume that there are nonzero $c_{1}, \ldots, c_{n}$ such that each $z_{\zeta}$ has a representation

$$
z_{\zeta}=\sum_{l=1}^{n} c_{l} e_{\beta(\zeta, l)}
$$

and for all $\zeta<\eta$ we have $\alpha^{*}<\beta(\zeta, 1)<\ldots<\beta(\zeta, n)<\beta(\eta, 0)<\ldots<\beta(\eta, n)$.

By applying Lemma 3 to the family $\left\langle\operatorname{supp}\left(z_{\zeta}\right): \zeta\left\langle\lambda^{+}\right\rangle\right.$and $\mu=\omega$, we find $B \in\left[\lambda^{+}\right]^{\omega}$ and $n^{*}<\omega$ such that (ii) from the conclusion of Lemma 3 holds.

Note that now $\left\langle A_{\beta} \backslash \lambda_{n^{*}}: \beta \in \bigcup_{\zeta \in B} \operatorname{dom}\left(z_{\zeta}\right)\right\rangle$ is a family of disjoint sets. Since also $\left\langle\operatorname{dom}\left(y_{\nu}\right): \nu<\lambda\right\rangle$ is a disjoint family and the $g_{\alpha}$ 's are one-to-one we may find $y_{\nu}$ such that for all $\beta \in \bigcup_{\zeta \in B} \operatorname{dom}\left(z_{\zeta}\right)$ we have $g_{\beta}\left(\operatorname{dom}\left(y_{\nu}\right)\right) \subseteq A_{\beta} \backslash \lambda_{n^{*}}$. But now not even $m_{\nu} \cdot n$ many vectors $z_{\zeta_{1}}, \ldots, z_{\zeta_{m_{\nu} n}}$, where each $\zeta_{i} \in B$, are orthogonal on $y_{\nu}$. For if this would be true, then $\left\langle b_{1} c_{1}, \ldots, b_{m_{\nu}} c_{n}\right\rangle \in F^{m_{\nu} n}$ would be a nontrivial solution of the equation $A x=0$ where $A$ is the $\left(m_{\nu} n \times m_{\nu} n\right)$-matrix

$$
\left(\begin{array}{ccc}
\Phi\left(e_{\alpha(\nu, 1)}, e_{\beta\left(\zeta_{1}, 1\right)}\right) & \ldots & \Phi\left(e_{\alpha\left(\nu, m_{\nu}\right)}, e_{\beta\left(\zeta_{1}, n\right)}\right) \\
\vdots & & \vdots \\
\Phi\left(e_{\alpha(\nu, 1)}, e_{\beta\left(\zeta_{m_{\nu} n}, 1\right)}\right) & \ldots & \Phi\left(e_{\alpha\left(\nu, m_{\nu}\right)}, e_{\beta\left(\zeta_{m_{\nu} n}, n\right)}\right)
\end{array}\right) .
$$

But by construction and Lemma 2, this matrix has a nonzero determinant, a contradiction.

By Lemma 1 in $\S 3$, the upper bound for a $\lambda$-Gross space over $F$ is $|F|^{\lambda}$. Hence in ZFC, the dimension of a space as in Theorem 5 cannot be enlarged. But the following theorem shows that it can be enlarged at the loss that we only obtain a $\lambda^{+}$-Gross space. An upper bound for a $\lambda^{+}$-Gross space over a field of size $\lambda$ is $\lambda^{\lambda^{+}}=2^{\lambda^{+}}$. So again, in ZFC we cannot expect a better result. Theorem 6 is true also for countable fields, so we get a complement to Theorem 1 .

The construction will use filtrations given by the following Lemma.

Lemma 4. Let $\lambda$ be a cardinal.

(A) There exists a family $\left\langle A_{\gamma}^{\alpha}: \gamma<\lambda^{+}, \alpha<\lambda^{++}\right\rangle$of sets of size $\leq \lambda$ such that for any $\alpha, \beta<\lambda^{++}$and $\gamma, \delta<\lambda^{+}$the following requirements are satisfied:

(1) $\left\langle A_{\gamma}^{\alpha}: \gamma<\lambda^{+}\right\rangle$is increasing, continuous such that $\alpha=\bigcup_{\gamma<\lambda^{+}} A_{\gamma}^{\alpha}$ and $A_{0}^{\alpha}=\emptyset$.

(2) if $\gamma<\delta$, then $A_{\gamma}^{\alpha} \subseteq A_{\delta}^{\alpha}$.

(3) if $\alpha<\beta$ and $\alpha \in A_{\gamma}^{\beta}$, then $A_{\gamma}^{\alpha}=A_{\gamma}^{\beta} \cap \alpha$.

(B) Let $\left\langle C_{\alpha}: \alpha<\lambda^{++}\right\rangle$be a family of clubs of $\lambda^{+}$. There exists a family $\left\langle C_{\alpha}^{\prime}: \alpha<\lambda^{++}\right\rangle$of clubs and a sequence $\left\langle A_{\gamma}^{\alpha}: \gamma \in C_{\alpha}^{\prime}, \alpha<\lambda^{++}\right\rangle$of sets of size $\leq \lambda$ such that for any $\alpha, \beta<\lambda^{++}$and $\gamma, \delta<\lambda^{+}$we have $C_{\alpha}^{\prime} \subseteq C_{\alpha}$, and if $\alpha<\beta$ the set $C_{\beta}^{\prime} \backslash C_{\alpha}^{\prime}$ is bounded and the following requirements are satisfied:

(1) $\left\langle A_{\gamma}^{\alpha}: \gamma \in C_{\alpha}^{\prime}\right\rangle$ is increasing, continuous such that $\alpha=\bigcup_{\gamma \in C_{\alpha}^{\prime}} A_{\gamma}^{\alpha}$ and $A_{0}^{\alpha}=\emptyset$.

(2) if $\gamma, \delta \in C_{\alpha}^{\prime}$ and $\gamma<\delta$, then $A_{\gamma}^{\alpha} \subseteq A_{\delta}^{\alpha}$.

(3) if $\alpha<\beta$ and $\alpha \in A_{\gamma}^{\beta}$, then $C_{\beta}^{\prime} \backslash C_{\alpha}^{\prime} \subseteq \gamma$ and $A_{\gamma}^{\alpha}=A_{\gamma}^{\beta} \cap \alpha$.

Proof. We prove only (A). The proof of (B) is similar.

Assume that $\left\langle A_{\gamma}^{\nu}: \gamma<\lambda^{+}\right\rangle$for $\nu<\alpha$ have been constructed satisfying the requirements. Let $\left\langle\alpha_{\zeta}: \zeta<\lambda^{+}\right\rangle$be an enumeration of $\alpha$. Then the set

$$
\left\{\gamma<\lambda^{+}:\left(\forall \zeta_{1}, \zeta_{2}<\lambda^{+}\right) \text {if } \zeta_{1}<\zeta_{2} \text { and } \alpha_{\zeta_{1}}<\alpha_{\zeta_{2}} \text { then } \alpha_{\zeta_{1}} \in A_{\gamma}^{\alpha_{\zeta_{2}}}\right\}
$$


clearly is a club of $\lambda^{+}$. Let $\left\langle\gamma_{\varepsilon}: \varepsilon<\lambda^{+}\right\rangle$be its increasing enumeration and define $A_{0}^{\alpha}=\emptyset$, and

$$
A_{\gamma}^{\alpha}=\bigcup_{\zeta<\gamma_{\varepsilon}} A_{\gamma}^{\alpha_{\zeta}}
$$

if and only if $\gamma_{\varepsilon}<\gamma \leq \gamma_{\varepsilon+1}$.

It is not difficult to see that this works.

Theorem 6. Let $\lambda$ be an infinite cardinal. $A \lambda^{+}$-Gross space of dimension $\lambda^{++}$ exists over every field of size $\lambda$.

Proof. The proof is divided into two cases:

Case 1. There exists a family $\left\langle C_{\alpha}: \alpha<\lambda^{++}\right\rangle$of clubs of $\lambda^{+}$such that for every club $C$ of $\lambda^{+}$, for some $\alpha$ and $\lambda^{+}$many $\gamma \in C$ we have $\min (C \backslash(\gamma+1)) \leq \min \left(C_{\alpha} \backslash(\gamma+1)\right)$. Notice that this is equivalent to the assumption that there exist $\lambda^{++}$clubs of $\lambda^{+}$ such that no club eventually gets inside all of them.

By Lemma 4(B), without loss of generality we may assume that for $\alpha<\beta$ the set $C_{\beta} \backslash C_{\alpha}$ is bounded in $\lambda^{+}$and we have families $\left\langle A_{\gamma}^{\alpha}: \gamma \in C_{\alpha}\right\rangle$ satisfying (1), (2) and $(3)$.

Now let $F$ be a field of size $\lambda$ and $E$ a vector space over $F$, spanned by a basis $\left\langle e_{\alpha}: \alpha<\lambda^{++}\right\rangle$.

On $E$, we will define a symmetric bilinear form $\Phi$ by induction on $\alpha<\lambda^{++}$, using the filtrations above. Assume that $\Phi\left(e_{\beta}, e_{\beta^{\prime}}\right)$ has been defined for $\beta \leq \beta^{\prime}<\alpha$ and $\Phi\left(e_{\beta}, e_{\alpha}\right)$ has been defined for $\beta \in A_{\gamma}^{\alpha}$.

Let $\delta$ be the successor of $\gamma$ in $C_{\alpha}$. If $A_{\delta}^{\alpha} \backslash A_{\gamma}^{\alpha}$ has size less than $\lambda$, then $\Phi\left(e_{\beta}, e_{\alpha}\right)$, for $\beta \in A_{\delta}^{\alpha} \backslash A_{\gamma}^{\alpha}$, and $\Phi\left(e_{\alpha}, e_{\alpha}\right)$ may be defined arbitrarily. Otherwise, choose oneto-one enumerations such that $F=\operatorname{ran}\left\langle a_{\nu}: \nu<\lambda\right\rangle, A_{\gamma}^{\alpha}=\operatorname{ran}\left\langle\zeta_{\nu}: \nu<\lambda\right\rangle$ and $A_{\delta}^{\alpha} \backslash A_{\gamma}^{\alpha}=\operatorname{ran}\left\langle\xi_{\nu}: \nu<\lambda\right\rangle$.

Assume that $\Phi\left(e_{\xi_{\nu^{\prime}}}, e_{\alpha}\right)$ has been defined for $\nu^{\prime}<\nu$.

Define $\Phi\left(e_{\xi_{\nu}}, e_{\alpha}\right)$ such that $\Phi(z, y) \neq 0$ whenever $z$ and $y$ are vectors such that $\alpha \in \operatorname{dom}(z) \subseteq\{\alpha\} \cup\left\{\zeta_{\nu^{\prime}}: \nu^{\prime}<\nu\right\}$ and $\xi_{\nu} \in \operatorname{dom}(y) \subseteq\left\{\xi_{\nu^{\prime}}: \nu^{\prime} \leq \nu\right\}$ and all coefficients of $z$ and $y$ are contained in $\left\{a_{\nu^{\prime}}: \nu^{\prime}<\nu\right\}$. There are less than $\lambda$ many such $z$ and $y$. Hence, this definition can be fulfilled.

We will show that $\langle E, \Phi\rangle$ is a $\lambda^{+}$-Gross space. Assume that this is not true. So there is a subspace $U \subseteq E$ of dimension $\lambda^{+}$, say $U=\operatorname{span}\left\langle y_{\nu}: \nu\left\langle\lambda^{+}\right\rangle\right.$, such that in $U^{\perp}$, there is a family of $\lambda^{++}$linearly independent vectors, say $\left\langle z_{\iota}: \iota<\lambda^{++}\right\rangle$.

Without loss of generality, we may assume that $\left\langle\operatorname{supp}\left(y_{\nu}\right): \nu\left\langle\lambda^{+}\right\rangle\right.$is a family of pairwise disjoint sets of the same cardinality and each $y_{\nu}$ has the same nonzero coefficients in its representation. Mutatis mutandis, we may assume the same for the $z_{\iota}$ 's.

Choose $\alpha^{*}<\lambda^{++}$such that $U \subseteq \operatorname{span}\left\langle e_{\alpha}: \alpha<\alpha^{*}\right\rangle$.

We may certainly find a club $C \subseteq C_{\alpha^{*}}$ such that, if $\gamma<\delta$ are successive members of $C$, then

$$
\left|\left\{\nu<\lambda^{+}: \operatorname{dom}\left(y_{\nu}\right) \subseteq A_{\delta}^{\alpha^{*}} \backslash A_{\gamma}^{\alpha^{*}}\right\}\right|=\lambda .
$$

Since we are in Case 1 there exists $\alpha_{C} \in\left(\alpha^{*}, \lambda^{++}\right)$such that for $\lambda^{+}$many $\gamma \in C$ we have $\min (C \backslash(\gamma+1)) \leq \min \left(C_{\alpha_{C}} \backslash(\gamma+1)\right)$. 
Now let $\iota<\lambda^{++}$such that $\operatorname{dom}\left(z_{\iota}\right) \nsubseteq \alpha_{C}$ and let $\alpha$ be the largest member of $\operatorname{dom}\left(z_{\iota}\right)$. Hence $\alpha \geq \alpha_{C}, C_{\alpha} \backslash C_{\alpha_{C}}$ is bounded, and hence $S=\{\gamma \in C$ : $\left.\min (C \backslash(\gamma+1)) \leq \min \left(C_{\alpha} \backslash(\gamma+1)\right)\right\}$ has size $\lambda^{+}$.

Now choose $\gamma^{\prime} \in S$ so large that, if $\gamma$ is the maximal element of $C_{\alpha}$ such that $\gamma \leq \gamma^{\prime}$, then

$$
\left\{\alpha^{*}\right\} \cup \operatorname{dom}\left(z_{\iota}\right) \backslash\{\alpha\} \subseteq A_{\gamma}^{\alpha} .
$$

Let $\delta$ be the successor of $\gamma$ in $C_{\alpha}$. So clearly $\min \left(C \backslash\left(\gamma^{\prime}+1\right)\right) \leq \delta$.

By construction of the filtrations $\left\langle A_{\nu}^{\alpha}: \nu \in C_{\alpha}\right\rangle$ and by (1) and (2) we know that

$$
\left|\left\{\nu<\lambda^{+}: \operatorname{dom}\left(y_{\nu}\right) \subseteq A_{\delta}^{\alpha} \backslash A_{\gamma}^{\alpha}\right\}\right|=\lambda .
$$

Now look at the definition of $\Phi\left(., e_{\alpha}\right)$ on $A_{\delta}^{\alpha} \backslash A_{\gamma}^{\alpha}$. Choose $\nu<\lambda$ and $\mu<\lambda^{+}$ such that

(i) the coefficients of $z_{\iota}$ and those of $y_{\mu}$ (and hence of any $y_{\mu^{\prime}}$ ) are contained in $\left\{a_{\nu^{\prime}}: \nu^{\prime}<\nu\right\}$

(ii) $\operatorname{dom}\left(z_{\iota}\right) \backslash\{\alpha\} \subseteq\left\{\zeta_{\nu^{\prime}}: \nu^{\prime}<\nu\right\}$, and

(iii) $\xi_{\nu} \in \operatorname{dom}\left(y_{\mu}\right)$ and $\operatorname{dom}\left(y_{\mu}\right) \backslash\left\{\xi_{\nu}\right\} \subseteq\left\{\xi_{\nu^{\prime}}: \nu^{\prime}<\nu\right\}$.

By (3), such a choice is possible. But now $\Phi\left(e_{\xi_{\nu}}, e_{\alpha}\right)$ was defined such that $\Phi\left(z_{\iota}, y_{\mu}\right) \neq 0$, a contradiction.

Case 2. There exists no family of clubs as in Case 1.

Let $\left\langle A_{\gamma}^{\alpha}: \gamma<\lambda^{+}, \alpha<\lambda^{++}\right\rangle$be as in Lemma $4(\mathrm{~A})$.

Let $f_{\alpha}: \lambda^{+} \rightarrow \lambda^{+}$be defined by $f_{\alpha}(\gamma)=$ o.t. $\left(A_{\gamma+1}^{\alpha}\right)$. Note that if $\alpha<\beta$, then $f_{\alpha}(\gamma)<f_{\beta}(\gamma)$ for every $\gamma$ so large that $\alpha \in A_{\gamma}^{\beta}$.

Let $C_{\alpha}=\left\{\gamma<\lambda^{+}: \gamma\right.$ is a limit ordinal and $\left.(\forall \delta<\gamma) f_{\alpha}(\delta)<\gamma\right\}$. Clearly $C_{\alpha}$ is a club.

Since $\left\langle C_{\alpha}: \alpha<\lambda^{++}\right\rangle$cannot serve for Case 1 , there exists a club $C$ of $\lambda^{+}$such that

Let $\left\langle\gamma_{\varepsilon}: \varepsilon<\lambda^{+}\right\rangle$be the increasing enumeration of $C \cup\{0\}$. Now define $f^{*}$ : $\lambda^{+} \rightarrow \lambda^{+}$by:

$$
f^{*}(\gamma)=\gamma_{\varepsilon+2} \text { if and only if } \gamma_{\varepsilon} \leq \gamma<\gamma_{\varepsilon+1} .
$$

Using the definition of $C_{\alpha}$ and (4), it is easy to see that

$$
\forall \alpha<\lambda^{++} \exists \gamma<\lambda^{+} \forall \delta \in\left(\gamma, \lambda^{+}\right)\left[f_{\alpha}(\gamma)<f^{*}(\gamma)\right]
$$

Now let $F$ be a field of size $\lambda$ and $E$ a vector space over $F$ of dimension $\lambda^{++}$, spanned by a basis $\left\langle e_{\alpha}: \alpha<\lambda^{++}\right\rangle$.

Let $\left\langle F_{\gamma}: \gamma<\lambda^{+}\right\rangle$be an almost disjoint family of subsets of $F$ (so each $F_{\gamma}$ has size $\lambda$ and $F_{\gamma} \cap F_{\delta}$ has size $<\lambda$ for any $\gamma \neq \delta$ ) such that:

in case $\lambda$ is uncountable, $\bigcup F_{\gamma}$ is a set of elements which are algebraically independent over the prime field of $F$.

in case $\lambda=\omega, \bigcup F_{\gamma}$ is a subset of $\operatorname{ran}\left\langle a_{n}: n<\omega\right\rangle$ where $\left\langle a_{n}: n\langle\omega\rangle\right.$ is a sequence as in Lemma 1.

For each $\gamma<\lambda^{+}$, let $\left\langle a_{\varepsilon, \zeta}^{\gamma}: \varepsilon<\zeta<f^{*}(\gamma)\right\rangle$ be a one-to-one enumeration of $F_{\gamma}$.

In difference to the previous constructions in this paper, here the angles $\Phi\left(e_{\alpha}, e_{\beta}\right)$ will be defined upwards, as follows. 
For $\alpha<\lambda^{++}$and $\gamma<\lambda^{+}$define

$$
W_{\gamma}^{\alpha}=\left\{\beta<\lambda^{++}: \alpha \in A_{\gamma+1}^{\beta} \backslash A_{\gamma}^{\beta}\right\} .
$$

So $\lambda^{++} \backslash \alpha=\bigcup_{\gamma<\lambda^{+}} W_{\gamma}^{\alpha}$, and this is a disjoint union.

Now let $\alpha<\beta<\lambda^{++}$. We define

$$
\Phi\left(e_{\alpha}, e_{\beta}\right)=a_{\text {o.t. }\left(A_{\gamma+1}^{\alpha}\right), \text { o.t. }\left(A_{\gamma+1}^{\beta}\right)}
$$

in case o.t. $\left(A_{\gamma+1}^{\beta}\right)<f^{*}(\gamma)$ where $\gamma$ is the uniquely determined ordinal such that $\beta \in W_{\gamma}^{\alpha}$. Note that o.t. $\left(A_{\gamma+1}^{\alpha}\right)<$ o.t. $\left(A_{\gamma+1}^{\beta}\right)$ in this case. Otherwise, $\Phi\left(e_{\alpha}, e_{\beta}\right)$ may be defined arbitrarily. Also $\Phi\left(e_{\alpha}, e_{\alpha}\right)$ may be defined arbitrarily.

We claim that $\langle E, \Phi\rangle$ is $\lambda^{+}$-Gross. If this is not true, then there is a subspace $U \subseteq E$ of dimension $\lambda^{+}$, say $U=\operatorname{span}\left\langle y_{\nu}: \nu<\lambda^{+}\right\rangle$, such that in $U^{\perp}$ there is a family of $\lambda^{++}$linearly independent vectors, say $\left\langle z_{\iota}: \iota<\lambda^{++}\right\rangle$.

Again, we may assume that $\left\langle\operatorname{supp}\left(y_{\nu}\right): \nu\left\langle\lambda^{+}\right\rangle\right.$is a family of pairwise disjoint sets of the same cardinality and each $y_{\nu}$ has the same nonzero coefficients in its representation. Mutatis mutandis, we may assume the same for the $z_{\iota}$ 's. We may also assume that each $\operatorname{supp}\left(z_{\iota}\right)$ is disjoint from $\alpha^{*}$ where $\alpha^{*}$ is chosen such that $U \subseteq \operatorname{span}\left\langle e_{\alpha}: \alpha<\alpha^{*}\right\rangle$. Let

$$
\begin{gathered}
y_{\nu}=\sum_{l=1}^{m} b_{l} e_{\alpha(\nu, l)}, \\
z_{\iota}=\sum_{l=1}^{n} c_{l} e_{\beta(\iota, l)} .
\end{gathered}
$$

By (5) and property $\mathrm{A}(3)$, Lemma 4, of the filtrations $\left\langle A_{\gamma}^{\alpha}: \gamma<\lambda^{+}, \alpha<\lambda^{++}\right\rangle$ we can choose $\gamma^{*}<\lambda^{+}$such that

(j) for each $\beta \in \bigcup_{\iota<\lambda} \operatorname{dom}\left(z_{\iota}\right)$ and $\gamma \in\left(\gamma^{*}, \lambda^{+}\right) f_{\beta}(\gamma)<f^{*}(\gamma)$, and

(jj) if $\beta_{1}, \beta_{2} \in \bigcup_{\iota<\lambda} \operatorname{dom}\left(z_{\iota}\right)$ and $\beta_{1}<\beta_{2}$, then $\beta_{1} \in A_{\gamma^{*}}^{\beta_{2}}$ and $\alpha^{*} \in A_{\gamma^{*}}^{\beta_{1}}$.

Now find $y_{\nu}$ such that $\operatorname{dom}\left(y_{\nu}\right) \cap A_{\gamma^{*}}^{\alpha^{*}}=\emptyset$. There are finitely many $\gamma_{1}, \ldots, \gamma_{p}>$ $\gamma^{*}$ such that

$$
\operatorname{dom}\left(y_{\nu}\right) \subseteq \bigcup_{i=1}^{p}\left(A_{\gamma_{i}+1}^{\alpha^{*}} \backslash A_{\gamma_{i}}^{\alpha^{*}}\right) .
$$

Then for all $\beta \in \bigcup_{\iota<\lambda} \operatorname{dom}\left(z_{\iota}\right)$ and $1 \leq i \leq p$ we have

$$
\operatorname{dom}\left(y_{\nu}\right) \cap\left(A_{\gamma_{i}+1}^{\alpha^{*}} \backslash A_{\gamma_{i}}^{\alpha^{*}}\right)=\operatorname{dom}\left(y_{\nu}\right) \cap\left(A_{\gamma_{i}+1}^{\beta} \backslash A_{\gamma_{i}}^{\beta}\right) .
$$

Call this set $M_{i}$. So for $\alpha \in M_{i}$ and $\beta \in \bigcup_{\iota<\lambda} \operatorname{dom}\left(z_{\iota}\right)$ we have $\beta \in W_{\gamma_{i}}^{\alpha}$ and hence, by $(\mathrm{j})$ above, $\Phi\left(e_{\alpha}, e_{\beta}\right)=a_{\text {o.t. }\left(A_{\gamma_{i}+1}^{\alpha}\right) \text {,o.t. }\left(A_{\gamma_{i}+1}^{\beta}\right)}^{\gamma_{i}} \in F_{\gamma_{i}}$. Notice that for distinct $\alpha, \alpha^{\prime} \in M_{i}$ we have o.t. $\left(A_{\gamma_{i}+1}^{\alpha}\right) \neq$ o.t. $\left(A_{\gamma_{i}+1}^{\alpha^{\prime}}\right)$, and for $\operatorname{distinct} \beta, \beta^{\prime} \in \bigcup_{\iota<\lambda} \operatorname{dom}\left(z_{\iota}\right)$ we have o.t. $\left(A_{\gamma_{i}+1}^{\beta}\right) \neq$ o.t. $\left(A_{\gamma_{i}+1}^{\beta^{\prime}}\right)$. Hence, by almost disjointness of the $F_{\gamma_{i}}$, we may find $\iota_{1}, \ldots, \iota_{m \cdot n}<\lambda$ such that for $1 \leq j \leq m n$ the sets

$$
\left\{a_{\text {o.t. }\left(A_{\gamma_{i}+1}^{\alpha}\right), \text { o.t. }\left(A_{\gamma_{i}+1}^{\beta\left(\iota_{j}, l\right)}\right)}^{\gamma_{i}}: \alpha \in M_{i}, 1 \leq i \leq p, 1 \leq l \leq n\right\}
$$


are pairwise disjoint and each of them contains $m \cdot n$ elements. If now

$$
\Phi\left(y_{\nu}, z_{\iota_{j}}\right)=\sum_{1 \leq l \leq m, 1 \leq k \leq n} b_{l} c_{k} \Phi\left(e_{\alpha(\nu, l)}, e_{\beta\left(\iota_{j}, k\right)}\right)=0
$$

would be true for every $1 \leq j \leq m n$, then $\left\langle b_{1} c_{1}, \ldots, b_{m} c_{n}\right\rangle \in F^{m n}$ would be a nontrivial solution of the equation $A x=0$ where $A$ is the $(m n \times m n)$-matrix

$$
\left(\begin{array}{ccc}
\Phi\left(e_{\alpha(\nu, 1)}, e_{\beta\left(l_{1}, 1\right)}\right) & \ldots & \Phi\left(e_{\alpha(\nu, m)}, e_{\beta\left(l_{1}, n\right)}\right) \\
\vdots & & \vdots \\
\Phi\left(e_{\alpha(\nu, 1)}, e_{\beta\left(l_{m n}, 1\right)}\right) & \ldots & \Phi\left(e_{\alpha(\nu, m)}, e_{\beta\left(l_{m n}, n\right)}\right)
\end{array}\right)
$$

But the rows of this matrix are the sets in (6), hence its determinant is nonzero, a contradiction.

\section{REFERENCES}

[B/S] B. Balcar and P. Simon, Cardinal invariants in Boolean spaces, General Topology and its Relations to Modern Analysis and Algebra V, Proc. Fifth Prague Topol. Symp. 1981 (J. Novak, ed.), Heldermann Verlag, Berlin, 1982, pp. 39-47. MR 84f:06024

[B] J. E. Baumgartner, Iterated forcing, Surveys of set theory, London Mathematical Society Lecture Note Series, no. 87 (A.R.D. Mathias, ed.), Cambridge University Press, Cambridge, 1983, pp. 1-59. MR 87c:03099

[B/Sp] J. E. Baumgartner and O. Spinas, Independence and consistency proofs in quadratic form theory, Journal of Symbolic Logic 57, no. 4 (1991), 1195-1211. MR 93b:11042

[B/G] W. Baur and H. Gross, Strange inner product spaces, Comment. Math. Helv. 52 (1977), 491-495. MR 56:15680

[B/Sh] A. Blass and S. Shelah, There may be simple $P_{\aleph_{1}}$ - and $P_{\aleph_{2}}$-points and the Rudin-Keisler ordering may be downward directed, Annals of Pure and Applied Logic 53 (1987), 213243. MR 88e:03073

[vD] E. K. van Douwen, The integers and topology, Handbook of set-theoretic topology (K. Kunen and J. E. Vaughan, eds.), North-Holland, Amsterdam, 1984, pp. 111-167. MR 87f:54008

[F] D. H. Fremlin, Cichon's diagram, Initiation à l'Analyse, Université Piere et Marie Curie, Paris, 1985.

[G] H. Gross, Quadratic forms in infinite dimensional vector spaces, Progress in Mathematics, vol. 1, Birkhäuser, Boston, 1979. MR 81f:10027

[G/O] H. Gross and E. Ogg, Quadratic spaces with few isometries, Comment. Math. Helv. 48 (1973), 511-519. MR 51:6360

[Go] M. Goldstern, Tools for your forcing construction, Set theory of the reals (H. Judah, ed.), Proceedings of the Bar Ilan Conference in honour of Abraham Fraenkel 1991, pp. 305-360. MR 94h:03102

[J] T. Jech, Set theory, Academic Press, New York, 1978. MR 80a:03062

[J/Sh] H. Judah and S. Shelah, Souslin forcing, Journal of Symbolic Logic 53, no. 4 (1988), 1188-1207. MR 90h:03035

[K] K. Kunen, Set theory. An introduction to independence proofs, North-Holland, Amsterdam, 1980. MR 82f:03001

[Sh1] S. Shelah, Proper forcing, Lecture Notes in Mathematics, vol. 942, Springer, 1982. MR 84h:03002

[Sh2] S. Shelah, On cardinal invariants of the continuum, Proceedings of the 6/83 Boulder conference in set theory (J. Baumgartner D. Martin and S. Shelah, eds.), Contemporary mathematics, vol. 31, AMS, 1984, pp. 183-207. MR 86g:03064

[Sh3] S. Shelah, Vive la difference I, Nonisomorphism of ultrapowers of countable models, in: Set theory of the continuum (H. Judah, W. Just, H. Woodin, eds.), Springer, New York, 1992, pp. 357-405. MR 94g:03068

[Sh4] S. Shelah, Strong negative partition relations below the continuum, Acta Math. Hungar. 58, no. 1-2 (1991), 95-100. MR 93d:03052 
[Sh5] S. Shelah, There are Jońsson algebras in many inaccessible cardinals, Cardinal Arithmetic, Oxford University Press, 1994.

[Sh6] S. Shelah, Further cardinal arithmetic, in press ([Sh430] in Shelah's list of publications), Israel Journal of Mathematics.

[Sh7] S. Shelah, Colouring and $\aleph_{2}$-c.c. not productive, in preparation ([Sh572] in Shelah's list of publications).

[Sp1] O. Spinas, Konsistenz- und Unabhängigkeitsresultate in der Theorie der quadratischen Formen, Dissertation, University of Zürich, 1989.

[Sp2] O. Spinas, Iterated forcing in quadratic form theory, Israel Journal of Mathematics 79 (1991), 297-315. MR 94k:03068

[Sp3] O. Spinas, An undecidability result in lattice theory, Abstracts of papers presented to the AMS 11, no. 2. (March 1990), 161.

[Sp4] O. Spinas, Cardinal invariants and quadratic forms, Set theory of the reals (H. Judah, ed.), Proceedings of the Bar Ilan Conference in honour of Abraham Fraenkel 1991, pp. 563-581. MR 94f:03059

Department of Mathematics, The Hebrew University of Jerusalem, Givat Ram, JERUSALEM, ISRAEL

E-mail address: shelah@math.huji.ac.il

Department of Mathematics, University of California, Irvine, California 92717

E-mail address: ospinas@math.uci.edu 